\title{
ACERCA DE RECURSOS Y PRÁCTICAS: APORTES PRELIMINARES RESPECTO DE LOS ALIMENTOS EN GUAYASCATE, PERÍODO COLONIAL TEMPRANO (SIGLOS XVI Y XVII). CÓRDOBA, ARGENTINA
}

\author{
TOWARDS PRACTICES AND RESOURCES: PRELIMINARY CONTRIBUTIONS \\ ABOUT FEEDING IN GUAYASCATE DURING XVI AND XVII. CÓRDOBA, \\ ARGENTINA
}

\author{
Romina Grana* y M. Laura López**
}

\begin{abstract}
El contacto entre nativos americanos y españoles durante la Colonia produjo grandes cambios en diferentes aspectos de los modos de vida de ambos grupos, y particularmente en la esfera alimenticia se introdujeron nuevas especies de vegetales con sus prácticas culinarias. El objetivo de este trabajo es reconocer los recursos alimenticios presentes en el paraje Guayascate (Córdoba, Argentina) durante el Período Colonial Temprano (siglos XVI y XVII) y trazar algunas referencias acerca de las prácticas asociadas a su ingesta. Partimos de la conjetura de que se produjo un mestizaje en la configuración de la dieta de modo tal, que los grupos incorporaron recursos o modos de procesamiento del "otro" sin discontinuar algunas prácticas culinarias. Se trata de un aporte que inicia las discusiones concernientes al tema para la región de estudio y que forma parte de los reconocimientos preliminares de los desarrollos culturales en la frontera sur de la Gobernación del Tucumán.
\end{abstract}

Palabras claves: Guayascate, Período Colonial, alimentos, prácticas.

The contact between Natives Americans and Spaniards during The Spanish Colony produced significant changes in different aspects of the ways of life of both groups, and particularly in the food sphere, new species of vegetables were introduced with their culinary practices. The paper aims to recognize the food resources presented in the Guayascate area (Córdoba, Argentina) during the Early Colonial Period (16th and 17th centuries) and to draw some associated practices. We conjecture there was a cultural mixture in the configuration of the diet in such a way that the groups incorporated resources and ways of food-processing the "other" without discontinuing some culinary practices. It is a contribution that initiates discussions on the subject for the study region and is part of the preliminary recognition of cultural developments in the southern border of the Government of Tucumán.

Key words: Guayascate, Colonial Period, foodstuffs, practices.

\section{Introducción}

El conocimiento respecto de la alimentación en el pasado colonial temprano proviene desde múltiples proxy y depende de las características de la región bajo estudio: cada espacio sociogeográfico es particular y habilita, por un lado, el análisis de aquello que está presente tanto en los documentos como en los registros arqueológicos y, por otro, la formulación de preguntas de aquello que no aparece en ninguna fuente de información (Pastor y Boixadós 2016). Indagar acerca de la alimentación habilita el reconocimiento acerca de si todos comen lo mismo y de la misma manera; con esto, pensamos a los alimentos y sus maneras de consumo como un vector de entrada a prácticas más amplias de la vida humana, sus rupturas, continuidades y modalidades de intercambios.

En este trabajo proponemos acercarnos a esta problemática entre quienes se asentaron en el paraje Guayascate, sitio arqueológico prehispánico y colonial (Cattáneo et al. 2013) emplazado en el área Norte de la actual provincia de Córdoba, Argentina, y que perteneció a la Gobernación del Tucumán, Virreinato del Perú, durante el Período Colonial Temprano (siglos XVI y XVII) (Bixio et al. 2010). Este sector sur de la Gobernación fue considerado marginal durante el proceso colonial

\footnotetext{
* IEH-Centro de Estudios Históricos "Prof. Carlos S.A. Segreti", Esc. de Letras, Facultad de Filosofía y Humanidades, Universidad Nacional de Córdoba. Miguel del Corro 308 (5000), Córdoba, Argentina.

Correo electrónico: rominagrana77@ffyh.unc.edu.ar

** CONICET-División Arqueología, Facultad de Ciencias Naturales y Museo, Universidad Nacional de La Plata. Laboratorio 129, Unidad Anexa al Museo, Av. 60 y 122 s/nº (1900), La Plata, Argentina. Correo electrónico: mllopezdepaoli@ gmail.com
} 
debido a su ubicación geográfica en la periferia del imperio español y la ausencia de recursos de alto valor (metales). Este paraje estaba ocupado por grupos nativos organizados bajo un sistema político, económico y social bien establecidos, que se vio alterado en función de los cambios que sucedieron tras la fundación de ciudades y el repartimiento de tierras (Tell 2019).

En relación con esto, la alimentación acompañó estas alteraciones no solo a los españoles que trataron de instaurar las costumbres culinarias europeas, sino también a los nativos que se vieron restringidos en el acceso a los recursos que formaban parte de su dieta. La disponibilidad de productos, las elecciones acerca de qué comer, cómo preparar los alimentos y con quién compartirlos (comensalismo) ${ }^{1}$ dependen en gran medida de los valores establecidos culturalmente, lo que dará significado a la reproducción social y marcará instancias de fuertes diferenciaciones: los alimentos son un factor de distinción de las prácticas de los sujetos en la medida en que, por ejemplo, visibilizan rutinas domésticas o instancias más solemnes, es decir, nos hablan de la extraordinariedad o no de lo que se dispone para llevar a la mesa (Twiss 2007); así, existe todo un sistema de prácticas asociadas a la alimentación, de modo tal que se puede hablar de un "proceso de identificación" con los que un grupo o sociedad "se recrea en cada nueva práctica de cocinar y comer" (Álvarez 2005:17) y, con esto, cada ingrediente es a la vez integrador e integrante de un todo en el que la comida y el factor social están al servicio de la construcción de identidades que se cruzan, se discriminan pero también se fusionan. En este sentido, es interesante advertir cómo el mundo de la alimentación autoriza a pensar, por un lado, en el acceso al universo patrimonial tangible e intangible (o simbólico) (Garaycochea 2016) de un grupo humano y, por otro, permite visibilizar la inclusión o rechazo de alimentos en la dieta. El arribo de los españoles en América implicó un fuerte impacto tanto ambiental como cultural (Crosby 2003), porque nuevas plantas y animales pasaron a conformar parte de las dinámicas productivas y alimenticias de los nativos. En esta coyuntura y siguiendo la propuesta de Gruzinski (2007) en relación con el mestizaje cultural surgido en el siglo XVI, entendemos que los nativos tuvieron una amplia flexibilidad para adaptarse a las nuevas circunstancias de producción y alimentación, sin descartar que fuese la única manera de sobrevivir: tal flexibilidad permitió una asimilación de los nuevos productos, pero su ingesta no habría alterado el significado cultural de la comida. No obstante, para acercarnos al sentido que estos cambios pudieron tener para los indígenas y los españoles en este proceso, debemos primero indagar en aquellos frutos y granos que ingresaron a la región de estudio y los modos de preparación para su ingesta.

El objetivo específico de este trabajo es reconocer los vegetales que circularon y que pudieron incorporarse a la dieta tanto de los españoles como de los indígenas a partir de la lectura de fuentes históricas y materiales producidos sobre/en Guayascate. Para ello se describirán los frutos y granos que figuran en los documentos y avalan los registros arqueológicos, recursos bajo producción como de recolección (silvestres). Asimismo, intentaremos delinear la posible ingesta de vegetales, el empleo de técnicas y preparaciones culinarias y, finalmente, arriesgaremos algunas hipótesis del posible significado de su consumo. Con esto se pretende iniciar la discusión pertinente a los cambios y las continuidades de las costumbres en lo referente a los recursos disponibles que integraban la dieta en esta región, discusión que ya fue iniciada para otras de Argentina (Marschoff 2014).

\section{Espacialidad, corpus documental $\mathrm{y}$ aspectos metodológicos}

La localidad de Guayascate se ubica en el departamento Tulumba, en el norte de la actual provincia de Córdoba, Argentina, a $160 \mathrm{~km}$ de la capital (Figura 1). A mediados del siglo XVI fue parte del extenso territorio de la Gobernación del Tucumán que, aún con límites geográficos imprecisos, comprendió a las actuales provincias de Jujuy, Salta, Tucumán, Santiago del Estero, Catamarca, La Rioja y Córdoba (Bixio y Berberián 2017). Emplazada a $900 \mathrm{~m}$ sobre el nivel del mar, esta zona pertenece al Distrito Chaqueño Serrano de la Provincia Fitogeográfica Chaqueña, según Cabrera (1976), con predominancia del bosque cerrado a semicerrado con 30 a $60 \%$ de cobertura arbórea (Giorgis 2017), muchas de ellas con importancia alimenticia (Saur Palmieri et al. 2018).

Guayascate fue mencionado por primera vez en la documentación hispana a fines del siglo XVI tras el otorgamiento de una Encomienda a Francisco Sánchez entre 1574 y 1576 AD (Montes 2008:266). Posteriormente, esta pasó a manos de la familia García: el primer nombre que recoge la 


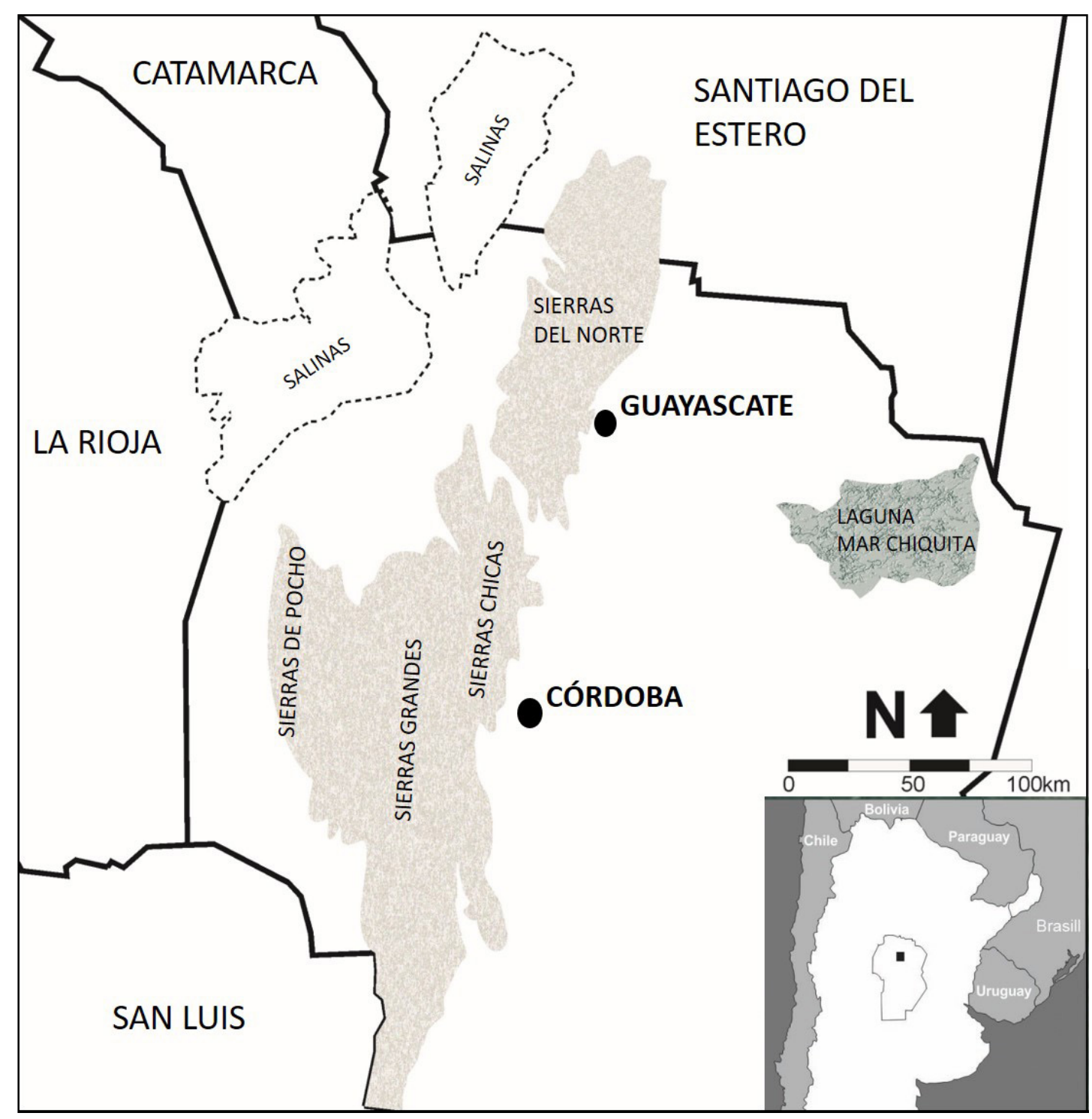

Figura 1. Mapa de ubicación de Guayascate en la actual provincia de Córdoba. Fuente: Autoría propia.

bibliografía es Bartolomé García, hijo de Pedro García y Francisca Núñez; suponemos que García hijo heredó la encomienda de su padre, pero acerca de estos datos no se explaya Montes (2008) ni hemos podido corroborarlos accediendo a fuentes directas pues están fuera de consulta por su notable deterioro. Si bien existen zonas de vacancia en esta génesis ( $v g$. Tiempo de tenencia de la encomienda), hay varias certezas que conviene mencionar:

- del matrimonio de Pedro García con Francisca Núñez nacieron, además de Bartolomé, otros dos hijos: Pedro y Nicolás García.
- Francisca Núñez enviudó, a finales del siglo XVI, y se casó en segundas nupcias con Juan de Torreblanca. Los documentos arrojan datos de la existencia de una hija de Francisca, Catalina González, casada con Juan Bernal de Mercado, pero no se aclara quién es su padre.

- Luego de la muerte de Pedro García padre, las posesiones pasan a su hijo Bartolomé quien es beneficiado con una Merced de tierra que le otorga el teniente de gobernador Juan de Burgos el 6 de abril de 1585: Bartolomé fallece en los albores del 1600, época en la que también muere su madre. 
La consulta de otras fuentes advierte la continuidad de la naturaleza conflictiva de las relaciones dentro del grupo español, así como también de las tensiones con los nativos:

- existe una demanda de los hijos de Francisca Núñez contra Torreblanca por los bienes dejados por su madre (AHPC, Esc. 1, 1605, Leg. 16, Exp. 4).

- existe la respuesta de Torreblanca a la demanda de los hijos García y el esposo de su hermana, Juan Bernal de Mercado (AHPC, Esc. 1, 1605, Leg. 18, Exp. 2). De este documento surgen datos relativos a las actuaciones de Torreblanca mientras administró los bienes de los menores.

- existe un pleito (AHPC, Esc.1, 1605, Leg. 16, Exp. 6) en donde Bernavé de Castañeda se querella contra los bienes y herederos de Bartolomé García difunto por cobro de pesos. En esta contienda se destaca el hecho de que quien se opone a la ejecución de los bienes es Torreblanca en función de defender especialmente el molino que, arguye, fue construido por él mismo.

- existe un expediente (AHPC, Esc. 1, 1611, Leg. 25, Exp. 4) destacable pues en su interior (fo.149r con fecha marzo 1610) se incluyen los autos de la demanda que presentan los indios Pedro Tumbaque, Rodrigo Anaca y Agustín Cama ante el Teniente de Gobernador Juan de Tejeda y Miraval; ellos reclaman su derecho a tierras que han sido adjudicadas a los herederos de Bartolomé García. En los folios finales se encuentra la sentencia del Teniente Gobernador en favor de los indios e incluso un texto producido por Alfaro el 4 de mayo de 1611 que convalida la sentencia, ampara a los indios y ordena la no ejecución en favor de los herederos del encomendero.

Utilizamos además otros escritos inéditos de la segunda mitad del siglo XVII, aunque existe un vacío de información para los años intermedios por la imposibilidad de su consulta: en 1670 se registra un testamento sucesorio de doña Lucía Martel de los Ríos, mujer de Juan/Julio de Olariaga quien fuera encomendero de Guayascate para esa fecha. En él se mencionan animales y vegetales (AHPC, Esc. 1, 1670, Leg. 133, Exp. 7).

Por último, analizamos una fuente édita de gran importancia: la visita que realizó el oidor de la
Audiencia de Charcas, don Antonio Martínez Luxán de Vargas a la jurisdicción de Córdoba (Bixio 2009). Para la fecha en que llegó el visitador (1692/1963), el encomendero de los indios de Guayascate era Leandro Ponze de León y, el cacique principal, Antonio Samaniego. En los autos para esta encomienda consta una clara descripción de los bienes y productos de Guayascate.

El trazado realizado cubre casi un siglo y nos permitió delinear la continuidad o no en la manipulación de algunos vegetales, así como también vislumbrar prácticas asociadas a ellos. Estas aportaciones fueron ampliadas con datos procedentes de investigaciones etnohistóricas (Borrastero 2021, Lorandi 1988, Doucet 1990, Piana 1992; entre otros) que contribuyeron a contextualizar los datos para ponerlos en perspectiva con aquellos provenientes del resto de la actual provincia cordobesa y de toda la Gobernación del Tucumán.

El tratamiento metodológico de las fuentes es de tipo cualitativo interpretativo: las consideraciones que ofrecemos son válidas para un abordaje que obliga a desplazar la atención del hecho histórico al documento histórico (Bixio y Berberián 2009). Así, la interpretación de los datos que presentamos fue habilitada a partir de ciertas preguntas que hicimos a las fuentes con lo que sostenemos que es el historiador el que, intencionalmente, construye, en la medida en que interroga, el documento: sus intervenciones respecto de la masa discursiva son las que operan la transformación: (...) en síntesis, el documento es una construcción, una elaboración del historiador que lo recorta, lo elige, lo organiza, lo reparte, lo inserta en series (...) (Foucault 1985:10).

Finalmente, el análisis se hace desde una perspectiva que integra conocimientos provenientes de disciplinas diversas orientadas a la indagación del mismo problema: las categorías y métodos de la historia, la arqueología, y el análisis sociohistórico de los discursos se cruzan y conviven en la medida en que la complejidad del problema abordado no puede ser indagado desde una única mirada.

\section{Los recursos disponibles. Producción y recolección}

Los documentos consultados han ofrecido información tanto de los frutos nativos de la región chaqueña serrana como también de aquellos endógenos y adventicios producidos en tierras de Guayascate antes y después del asentamiento español 
(Tabla 1). Es posible recordar que, en la Jurisdicción de Córdoba, el otorgamiento de Mercedes de tierras no implicó la desaparición de las Encomiendas y es por ello, por lo que se puede observar, que el pago de tributos por parte de los indígenas se dio como servicio personal, trabajando para las actividades económicas del encomendero bajo la figura del libre concierto (Borrastero 2021, González Navarro 2010).

\section{Cultivos}

\section{Viñas}

Las referencias concretas y más tempranas que hemos podido recabar de cultivos son de 1605 (AHPC Esc. 1, 1605, Leg. 16, Exp.6) en el pleito por la ejecución de los bienes de Bartolomé García, entre los que se encuentran viñas (Vitis sp.), referencia que posteriormente se reitera: Melchor González Paral, testigo, dice que "de nueve años a esta parte conocio en las tierras de Guayascate a Bartolome Garcia encomendero de los indios que allí tenia y andava el dicho Bartolome Garcia plantando una viña..." (AHPC Esc. 1, 1611, Leg. 25, Exp. 4, f. 170v) y, un poco antes, encontramos que el encomendero "tiene en el dicho sitio echos viñas molino huertas y arboleda" (AHPC Esc. 1, 1611, Leg.25, Exp.4, f.146r). A partir de estas referencias es posible pensar que las plantas fueran pequeñas, con lo que estaríamos frente a una zona de cultivo relativamente nuevo en el lugar. Abundan en este expediente las menciones a las viñas en boca de todo tipo de locutores quienes ratifican la existencia de este cultivo.

La uva aparece como uno de los recursos hispanos en la región desde el inicio de la encomienda hasta, por lo menos, finales del siglo XVII. Consideramos que los datos se corresponderían con la producción de Vitis vinifera, especie traída desde Europa a partir del segundo viaje de Cristóbal Colón. Tras su éxito en las plantaciones de Perú, fue distribuida a Chile y luego a Argentina para la segunda mitad del siglo XVI (Carbonari y Cobo 2013). No existe por el momento evidencia arqueológica en la Gobernación del Tucumán, pero sí se registra su presencia en los documentos (Castillón 2021).

Es interesante observar la existencia de viñas en Guayascate a pesar de la prohibición de las plantaciones de viñas, vides y la producción y comercialización de vino en el Virreinato del Perú -situación que se repite en todo el territorio sudamericano (Lara et al. 2006)- cuyo circuito

Tabla 1. Recursos vegetales registrados en la documentación analizada

\begin{tabular}{|c|c|c|c|c|c|}
\hline $\begin{array}{c}\text { Registro de recursos } \\
\text { vegetales }\end{array}$ & $\begin{array}{l}\text { Nombre científico } \\
\text { identificado }\end{array}$ & Proveniencia & Estatus & Usos & $\begin{array}{l}\text { Práctica culinaria } \\
\text { registrada }\end{array}$ \\
\hline Viñas & $\begin{array}{l}\text { Vitis sp. (posiblemente } \\
\text { V. vinifera) }\end{array}$ & Europa & Doméstica cultivada & Alimenticio & Sin datos \\
\hline Trigo & Triticum sp. & Europa & Doméstica cultivada & Alimenticio & Producción de harina. \\
\hline Cebada & Hordeum vulgare & Europa & Doméstica cultivada & Posible forraje & Sin datos \\
\hline Maíz & Zea mays & América & Doméstica cultivada & Alimenticio & $\begin{array}{l}\text { Molienda. } \\
\text { Bebida fermentada } \\
\text { (chicha) }\end{array}$ \\
\hline Algarroba & $\begin{array}{l}\text { Prosopis sp. (posible- } \\
\text { mente } P \text {. alba, } \text { P. nigra, } \\
\text { P. chilensis). }\end{array}$ & América & Silvestre no cultivada & Alimenticia & $\begin{array}{l}\text { Comidas. Bebida } \\
\text { fermentada. }\end{array}$ \\
\hline Árboles / Arboleda ${ }^{2}$ & Sin datos específicos & $\begin{array}{l}\text { Sin datos } \\
\text { (posible } \\
\text { Europa) }\end{array}$ & Cultivadas (fruticultura) & Alimenticias & Sin datos \\
\hline Heredades $^{2}$ & Posible Olea europea & Euroasiático & $\begin{array}{l}\text { Cultivada } \\
\text { (fruticultura) }\end{array}$ & $\begin{array}{l}\text { Alimenticias y po- } \\
\text { sible utilitarias }\end{array}$ & Sin datos \\
\hline Huertas & Sin datos específicos & Sin datos & Cultivada & Alimenticia & Sin datos \\
\hline
\end{tabular}

${ }^{1}$ Si bien en la documentación solo se menciona árboles/arboledas, Castillón (2021) en su trabajo referente a las especies que pueden ser incluidas bajo este término en los documentos del siglo XVI y XVII en otras jurisdicciones de la Gobernación del Tucumán, menciona árboles frutales provenientes de Europa.

${ }^{2}$ Heredades hace referencia en el siglo XVI-XVII al olivar o la vid indistintamente, a menos que se mencione uno de esos cultivos de manera separada (Gamero Rojas 2011) como es nuestro caso: "allí han traido parte de sus indios para hazer sementeras e plantar heredades viñas e otros arboles que poseen” (Escr 1; 1611, Leg. 25, exp. 4, f186v). 
mercantil se inició hacia finales del siglo XVI y fue afianzándose en el Corregimiento de Cuyo (Gobernación de Chile) donde Córdoba era un nexo importante en la distribución hacia el Tucumán y Buenos Aires (Rivera Medina 2006). No es clara la finalidad de estas plantaciones: se tiende a pensar, por documentación de la región, que pudo destinarse a la comercialización o producción de vino, ya que en cercanías a Guayascate, en las actuales localidades de Jesús María y Colonia Caroya, los jesuitas produjeron vino (Domínguez y Heredia s/f).

Si las conjeturas de producción vinícola fueran acertadas, podríamos presumir que su consumo era español, ya que los nativos siguen elaborando "chicha" (ver más adelante). Asimismo, podríamos suponer su empleo en las misas para evangelizar a los nativos. No obstante, su destino aún es incierto.

\section{Trigo, maíz y cebada}

El trigo (Triticum sp.) y el maíz (Zea mays) también fueron registrados y encontramos que, en 1611, Andrés de Ceballos, testigo, dice "que sabe e vido (...) que Bartolome Garcia (...) labraba y cultivaba las dichas tierras haciendo sementeras de trigo y mais con los indios" (AHPC Esc. 1, 1611, Leg. 25, Exp. 4, f. 182r). Hacia 1670, en el testamento de Lucía Martel de los Ríos se mencionan, entre los bienes testados, "diez fanegas de harina" (AHPC Esc.1, 1670, Leg.133, Exp.7, F.99).

Trigo y maíz aparecen con relativa frecuencia en la documentación de las primeras décadas del siglo XVII y reaparecen con importante visibilidad en los autos de la Visita de Luxán de Vargas; a ellos se suma la presencia de cebada (Hordeum vulgare), lo que se puede corroborar en el siguiente extracto en el que hay una especie de recuento de las semillas para finales del 1600:

"El capitan Juan Lopez de Fuenteseca rexidor y protector general de los naturales (...) del pueblo de Guayascate (...) digo (...) porque la obligazion que tiene cada yndio de tassa conforme a la hordenanza 97 ademas de los çinco pesos de tributo en plata o, en especie que a de pagar de que a de sembrar en sus propias tierras, chacras, de comunidad, a razon de quatro almudes de trigo, dos de zeuada, y un quartillo de maiz" (Bixio 2009:f.28r).
Otros pasajes de la Visita corroboran la misma idea, así, Joseph Labastra -testigo- responde a la pregunta de si reciben doctrina y oyen misa diciendo que "dicho pueblo a tenido capilla que es la que esta en frente de la ramada (testado: que) es que esta el dicho señor Visitador que al presente se halla caida por la humedad que reciuio del riego que se dio al trigo que sembro hasta la mesma capilla el dicho encomendero" (Bixio 2009:f.6v).

El maíz es la especie americana más cultivada en toda América (Pochettino 2015). Los hallazgos arqueológicos en el Noroeste Argentino (NOA) son reflejo de la actividad prehispánica cotidiana como ritual, tanto doméstica como comunitaria (Korstanje 2015; Lema 2014). El maíz arribó a la región central argentina entre el 1000-500 AD, y se cultivó desde el Período Prehispánico Tardío (400-1550 AD) como parte del sistema económico de las poblaciones nativas y contribuyó al desarrollo de una horticultura de baja tecnificación basada en la conjunción del maíz, porotos (Phaseolus sp.) y zapallo (Cucurbita sp.) (Pastor y López 2011); todo acorde a un sistema de asentamiento y subsistencia de grupos dispersos, de economía mixta (caza-recolección-producción) con conexiones de parentesco entre los diferentes grupos, que se reunían esporádicamente por razones políticas, sociales o económicas (Medina et al. 2016).

Esta especie ha sido recuperada en sitios del sector serrano, lo que sirve para dar cuenta de su producción, procesamiento y consumo (López 2018). En Guayascate, el maíz fue registrado en cuencos cerámicos de características prehispánicas, lo que es signo de su manipulación (López 2020), pero sin un contexto de procedencia claro. Son pocos los sitios del Tucumán donde se han recuperado restos de maíz en contexto colonial (Capparelli et al. 2005; Petrucci et al. 2018), de entre ellos sobresale en Córdoba el sitio Alero Tala Huasi (Depto. Punilla) (López 2018; Pastor y Medina 2013). No obstante, podemos observar que no solo se integraba como comida sino también como bebida fermentada, ya que se reducía "a chicha que de ello hazen para sus embriaguezes con que con solo la sementera que hazen para su encomendero aseguran en ella la carne y el grano para comer y beuer" (Bixio 2009:f.24v).

Podemos afirmar que su consumo fue continuo entre los nativos: lo cultivaban para sí, para el encomendero y podían recibirlo como parte de pago por servicio prestado (Borrastero 2021, 
González Navarro 2010). Asimismo, es posible pensar que el encomendero no lo consumiera, sino que lo utilizara como alimento para sus animales o bien lo integrara a algún circuito mercantil (Piana 1992). Los estudios realizados en España acerca de la incorporación de los vegetales comestibles americanos en la dieta española demuestran que existió una primera etapa de rechazo al maíz y luego pasó a cultivarse como alimento para animales de granja, vacadas y los estamentos más pobres de la sociedad (Paz Sánchez 2013).

El maíz parece representar el único recurso americano cultivado que se mantiene en producción desde la ocupación española en tierras de Guayascate. Podemos pensar que para los nativos fue fuente no solo de alimentación sino también de reconocimiento de una identidad compartida que los separa de los extranjeros que posiblemente no lo consumieran. La elaboración de comidas y bebidas no solo habría representado un medio de subsistencia sino también parte del comensalismo grupal, remarcando símbolos indígenas impresos en dicho recurso y su modo de ingesta.

La presencia de trigo y cebada en Argentina, gramíneas euroasiáticas que se cultivan principalmente para producir harina (Pochettino 2015), se registra a partir del siglo XVI. Capparelli et al. (2005) resumen las tres posibles rutas de acceso de estas al NOA a partir de la documentación histórica disponible. La primera ruta fue por el Océano Atlántico Sur en la segunda mitad del siglo XVI cuando los españoles se instalaron en la actual Buenos Aires. La segunda ruta unía Chile con Santiago del Estero, capital de la Gobernación en 1553; esta vía permitió que la ciudad se abasteciera de trigo y cebada desde 1556 en adelante debido a su escasez. La tercera ruta involucra a los grupos Huarpes, nativos de Mendoza, y los aborígenes de Chile, cuyas rutas de intercambios favorecieron la entrada de estos cultivos a Argentina entre 1543 y 1560.

Las evidencias arqueológicas de trigo y cebada en la Gobernación del Tucumán se reducen a restos recuperados en contextos domésticos y rituales comunitarios como productivos (Angiorama et al. 2018; Capparelli et al. 2005; Petrucci et al. 2018). En Córdoba solo hay menciones en el registro documental que muestran que los indios produjeron estas especies. Para ello el encomendero proveía las semillas y los implementos para la siembra, lo que, sin duda, implicó que los indios debieran aprender nuevas técnicas como es la utilización de bueyes: en la declaración de Rui de Sosa (AHPC Esc. 1, 1611, Le.25, Exp.4, f.176r) consta que "los indios siembran junto a las chacaras de su amo y a la redonda della para sustentarse los indios y que esto les dava su amo bueyes y adereço para podello hazer e de allí comían los indios de las dichas sementeras" y, más avanzado el siglo, en la Visita de Vargas, registramos que para sembrar están "obligados los yndios a poner solo su trauajo y tierras y los encomenderos, bueyes arados semilla, y demas aperos" (Bixio 2009:f.29r).

Es importante mencionar que, desde la fundación de Córdoba en 1573, esta región fue productora de trigo y se elaboraban sus derivados (harina), lo que explicaría la presencia del molino en Guayascate tal como consta en los registros de 1605 y 1611 que hemos mencionado. Con posterioridad a este primer decenio del siglo XVII la producción triguera decayó derivándose hacia la ganadería con reorientación a la comercialización hacia Potosí (Palomeque 2005). Esta situación podría explicar la incorporación más tardía de la siembra de cebada porque uno de sus usos habituales es como forraje (Pochettino 2015).

El trigo habría sido reducido a harina en el molino propio desde inicios del siglo XVII. A los datos de su presencia (AHPC Esc.1, 1670, Leg.133, Exp.7, f.99) se adiciona el dato indirecto que refiere al pan; decimos indirecto porque no hay referencias explícitas a su manufactura y no podemos asegurar si con "pan" se hace referencia a la masa de harina de trigo cocida, la que era consumida por los españoles, o si se trata del genérico de "alimento":

"El capitan don Leandro Ponze de Leon vesino encomendero de los yndios del pueblo de Guaiascate (...) digo que hallandome al presente con una sementera de trigo considerable y totalmente falto de jente para recojerlo (...) y se deue especialmente atender que no se malogren dichas sementeras de trigo para este año proximo venidero por lo que el presente se a padesido de hambre que aun a faltado el pan a las personas de mayor posibilidad y totalmente a los conbentos de las monjas de uno y otro monasterio" (Bixio 2009:f.27r).

El consumo de trigo por los indios aún no es claro; hay testimonios que ratifican que el encomendero les pagaba con ello, pero también se dice que los nativos no tenían tiempo ni tierras suficientes 
para cultivar sus comidas (maíz y demás especies nativas) (González Navarro 2008). No obstante, surge en la actual provincia de La Rioja, región oeste de la Gobernación del Tucumán, la mención a la participación de los indios en el circuito comercial mediante la intervención del encomendero; de esta manera, intercambiaban productos europeos obtenidos como medio de pago por sus servicios por otros productos como maíz (Giovannetti 2005). Para Guayascate no podemos asegurar esta misma situación por falta de evidencias. Sin embargo, la incorporación dentro de la dieta indígena, sea voluntaria o no, no puede descartarse, si se piensa que incorporaron la carne vacuna en reemplazo de la carne de caza, adecuándola a sus costumbres culinarias (Grana y López 2021). Si consideramos que la cultura gastronómica va más allá de los ingredientes y atraviesa los sentidos que se dan a la elaboración (Espeitx 2004) y pensando en la flexibilidad propia de los grupos nativos (Gruzinski 2007), el trigo pudo ser preparado al modo acostumbrado como resulta de la siguiente cita de Vargas: "y aun distribuidas sus raziones en cada semana las mas vezes dejan de comer el trigo ô mays por reduzirlo a chicha que de ello hazen para sus embriagueces" (Bixio 2009: f.24r). La producción de bebidas fermentadas (chicha) con maíz posee datos arqueológicos y etnobotánicos en todo el NOA (Pochettino 2015). Es destacable aquí el valor cultural de tal bebida alcohólica en el comensalismo entre los nativos para su reproducción social, y así como se menciona en la introducción, el valor en sí no es del ingrediente aislado sino del producto: fermentar trigo para obtener la bebida que luego se comparte constituiría un mecanismo de adaptación a la nueva situación colonial que no altera el sentido último que los comensales otorgan al hecho de fomentar la reunión y dinamizar sus intercambios sociales.

\section{Silvestres}

La planta silvestre nativa que se menciona es el algarrobo, una leguminosa arbórea que hace referencia al género Prosopis. A lo largo de la historia, se refleja su aprovechamiento con múltiples propósitos (Pochettino 2015). El consumo de su fruto, denominado "algarroba", es fresco o con procesamientos para obtener harina y luego mezclada con otros ingredientes o como patay (harina compactada y secada al sol), arrope o jalea (hervido de los frutos), aloja y añapa (ambas bebidas en distintos niveles de fermentación) (Saur Palmieri et al. 2018).

A nivel arqueológico, hay registro de Prosopis en todo el NOA desde aproximadamente el 2000 AC hasta la llegada de los españoles (Babot 2011, Capparelli 2008, Capparelli y Lema 2011, entre otros). En cuanto a los documentos, citas claves para la región como la de Sotelo de Narváez en su relación de la Provincia del Tucumán del siglo XVI, cronista que da cuenta acerca de los nativos de Córdoba, menciona: "No tenían fruta mas que cardones diferentes, tunas y algarroba y chañar" (Bixio y Berberián 2017:269), todo ello conduce a pensar que el algarrobo fue el alimento silvestre por excelencia de toda la población nativa del Tucumán. Sin embargo, no parece transmitirse tal relevancia a los pobladores del sector serrano cordobés, donde su consumo se combinaba con otros recursos arbóreos de importancia nutritiva como el chañar y el mistol, los que formaron un trío de disponibilidad anual variable, pero de colecta segura al menos de uno de ellos (López 2018).

En Guayascate los análisis de microrrestos botánicos demostraron su consumo, al menos, hasta la llegada de los españoles (López 2020). A esta evidencia arqueológica se suman los aportes documentales que permiten afirmar la existencia de este fruto en los primeros decenios posteriores a la colonización en zonas aledañas: en el pleito de 1611, Francisco Mexia afirma que

"los indios de Guayascate son naturales de Eschilin ques ocho leguas tienen tierras donde cultivaban antiguamente y algarrobales con que se sustentaban e ansy mesmo ay indios de Acampis pocos o de Mocacat los quales dichos indios Mocacapes tienen buenas tierras e algarrobales con que sustentaban en sus tierras" (f.186r).

Si bien el discurso de Mexia no tiene como tópico Guayascate sino sus alrededores (Ischilin), es posible inferir que el paisaje acusara también la presencia de algarrobales, pues abarca el área fitogeográfica de su distribución natural (Cabido y Zak 1999). Extendemos por tal esta consideración, pues la proximidad en el espacio de ambos lugares así la habilita.

Más de medio siglo después se menciona también este fruto cuando el Visitador dice respecto de 
las tareas que debían desempeñar los indios que "se dispuso en la real hordenanza treinta y dos combendria fuesen visitados los yndios por sus encomenderos señalandoles destos los tiempos en que pudiesen assistir en dichos pueblos como es el de sembrar deserbar y coger las comidas y el de coger la algarroba" (Bixio 2009: f.24v) y, más adelante, en f.28v se retoma la misma idea. En virtud de esto, afirmamos que se trata de un indicador de visibilidad del modo en que pudo haber sido incorporada a la dieta de quienes habitaban la sociorregión hacia finales del siglo XVII e incluso, si observamos otros aportes etnohistóri$\cos$, se sabe que la algarroba suele asociarse a las reuniones de comensalismo denominadas "juntas y borracheras" (Castro Olañeta 2006:153), donde los nativos interactuaban y reproducían prácticas culturales bajo árboles de algarrobo posiblemente al inicio de su recolección. Al igual que el maíz, el algarrobo y sus prácticas asociadas pudo constituir una forma de representación identitaria que continuaron reproduciendo bajo simbolismos que los españoles probablemente no pudieron entender o reconocer como tal.

No hay indicios del consumo por parte de los españoles ni tampoco datos que permitan inferir que el algarrobo haya formado parte del tributo otorgado al encomendero como consta para el NOA teniendo en cuenta las Ordenanzas de Abreu y de Alfaro (Noli 1999).

\section{Conclusiones preliminares: certezas para seguir indagando}

En términos generales, los recursos vegetales cuyos datos emanan del análisis documental, con apoyo de algunos datos arqueológicos, reflejan cierta continuidad en la producción de trigo, maíz y recolección de algarroba desde los primeros decenios posteriores a la conquista hasta finales de siglo XVII.

La vid pareciera haber tenido un momento de apogeo a comienzos del 1600 mientras que a fines de este siglo XVII solo hay una mención para otra localidad (el Pueblo de Pichana). En Guayascate no quedan registros de vides, con lo que es admisible hipotetizar su posible abandono ¿temporario/ definitivo?

El trigo y la cebada fueron producidos y luego pudieron tener un doble destino, sea la comercialización de los granos y de la harina o el consumo de estos por parte de los españoles y probablemente los grupos nativos, aunque no existen evidencias concretas. La harina, producida en el propio molino de Guayascate, pudo ser para elaborar, aspecto culinario que por el momento no podemos corroborar ni desestimar.

Específicamente respecto del maíz hay registros que permiten afirmar que era consumido como comida y bebida, continuando con técnicas culinarias nativas como la fermentación. Esta última revela otras prácticas culturales como el comensalismo del producto resultante, lo que es un tópico bastante recurrente en Actas Capitulares donde se intenta limitar su circulación para evitar las libertades y desenfrenos que su consumo ocasionaba.

En relación con el algarrobo, se puede vislumbrar la continuidad de la recolección, pero no podemos asegurar que fuese al mismo ritmo acostumbrado antes de la Colonia, ya que, si nos basamos en la falta de tiempo para recolectar y sembrar que declaran sufrir, lo más probable es que esta no haya sido sistemática.

En virtud de estos reconocimientos, sabemos cuáles son los recursos vegetales que circulaban en la región entre los siglos XVI y XVII desde la llegada de los españoles y también hemos podido advertir algunas prácticas asociadas a ellos. En función de las posibles extensiones analíticas que pudiéramos seguir desarrollando, es interesante reconocer que estas prácticas hablan de ciertos mecanismos de cohesión social que se ven favorecidos por la continuidad de reproducción de prácticas culinarias nativas. En este sentido, ofrecimos un primer rastreo de las condiciones que posibilitaron la vida y la apropiación del espacio en Guayascate antes que conclusiones definitivas que funcionan para la generación de nuevos interrogantes.

\section{Agradecimientos}

Agradecemos a la Dra. Beatriz Bixio por facilitarnos una transcripción aproximada del expediente de 1611. A la Lic. Noelia Silvetti por su generosidad al ofrecer las fotografías del pleito de 1605. A la Ing. Agrónoma Daniela Mansilla, quien aportó un recurso bibliográfico de gran interés para este trabajo. A los evaluadores anónimos cuyos comentarios permitieron enriquecer el manuscrito original. Esta investigación recibió financiación de CONICET (PIP 2014-2016 GI) y Programa de Incentivos UNLP (Ref. 11/N734), ambos dirigidos por la Dra. A. Capparelli. 


\section{Referencias Citadas}

Álvarez, M.

2005 "La cocina como patrimonio (in)tangible". En Primeras Jornadas de Patrimonio Gastronómico "La cocina como patrimonio (in)tangible”, editado por V. Mariani, pp. 11-26. Comisión para la Preservación del Patrimonio Histórico Cultural de la Ciudad de Buenos Aires, Buenos Aires,

Angiorama, C., Coronel, A. y M. Pignoni

2018 "Agricultura a pequeña escala durante tiempos prehispánicos y coloniales en Chajarahuayco 25 (Puna de Jujuy, Argentina)". Revista de Arqueología Histórica y Latinoamericana 12:12-36.

Babot, M.P.

2011 "Cazadores-recolectores de los Andes centro-sur y procesamiento vegetal. Una discusión desde la Puna Meridional Argentina (ca. 7000-3200 años AP)". Chungara 43(1): 413-432.

Bixio, B. y B. Berberián

2009 "Principios de análisis documental en arqueología". Xата (19-23):321-334.

Bixio, B. y E. Berberián

2017 Crónicas y Relaciones sobre el Antiguo Tucumán del siglo XVI (Córdoba-La Rioja-Santiago del Estero-TucumánCatamarca-Salta-Jujuy). Documentos y Estudios críticos. Editorial Brujas. Córdoba.

Bixio. B. y C. González Navarro

2009 "Dominación, Resistencia y Autonomía en el Extremo Sur del Virreinato del Perú (Siglos XVI y XVII)". Diálogos 13(2): 371-399.

Bixio, B., E. Berberián y S. Pastor

2010 Historia prehispánica de Córdoba. Editorial Brujas. Córdoba.

Bixio, B. (dir.).

2009 Transcripción y estudio de la visita de Antonio Martines Luxan de Vargas. Serie documental ${ }^{\circ}$ XVI. CEH Carlos Segreti. Editorial Brujas. Córdoba.

Borrastero, L.

2021 "Participación indígena en las actividades productivas del espacio rural de Córdoba. Revisión del pueblo de encomienda del Quilpo a fines del siglo XVI", en Diálogo Andino $\mathrm{N}^{\circ}$ 64:47-69.

Cabido, M. y M. Zak

1999 Vegetación del Norte de Córdoba. Secret. Agric., Ganad. y Rec. Renov. Prov. Cba. Córdoba.

Cabrera, A.

1976 "Regiones fitogeográficas argentinas". En Enciclopedia argentina de agricultura y jardinería, editado por W. Kugler, tomo 2, pp. 1-2. Acme, Buenos Aires.

Capparelli, A.

2008 'Caracterización cuantitativa de productos intermedios y residuos derivados de alimentos del algarrobo (Prosopis flexuosa DC y P. chilensis (Mol.) Stuntz, Fabaceae): aproximación experimental aplicada a restos arqueobotánicos desecados". Darwiniana 46(2):175-201.

Capparelli, A. y V. Lema

2011 "Recognition of post-harvest processing of algarrobo (Prosopis spp.) as food from two sites of Northwestern Argentina: an ethnobotanical and experimental approach for desiccated macroremains". Archaeol Anthropol Sci 3:71-92.
Capparelli A, Lema V, Giovannetti M y R. Raffino

2005 "The introduction of old world crops (wheat, barley and peach) in Andean Argentina during the 16th century AD: archaeobotanical and ethnohistorical evidence". Veg Hist Archaeobot 14:472-484.

Carbonari S. y A. Cobo

2013 La cultura de la vid y el vino: la vitivinicultura hace Escuela. XIV Jornadas Interescuelas/Departamentos de Historia. Facultad de Filosofía y Letras. Universidad Nacional de Cuyo, Mendoza.

Castillón, V.

2021 "Viñas y árboles frutales en el registro etnohistórico del noroeste argentino: apuntes para la investigación arqueológica". Chungara 53. En prensa.

Castro Olañeta, I.

2006 Transformaciones y continuidades de sociedades indígenas en el sistema colonial. El pueblo de indios de Quilino a principios del siglo XVII. Alción Editora, Córdoba.

Cattáneo, R., A. Izeta y T. Costa

2013 El patrimonio arqueológico de los espacios rurales de la provincia de Córdoba. Universidad Nacional de Córdoba. Córdoba.

Crosby, A.

2003 The Columbian Exchange. PRAEGER. Westport.

Doucet, G.

1990 "La encomienda de servicio personal en el Tucumán, bajo régimen legal: comentarios a las Ordenanzas de Gonzalo de Abreu". En Levaggi, A. (coord.). El Aborigen y el Derecho en el Pasado y el Presente. Buenos Aires, Universidad del Museo Social Argentino.

Espeitx, E.

2004 "Patrimonio alimentario y turismo: una relación singular". PASOS 2(2): 193-213.

Domínguez, I.M.B y T.B. Heredia s/f

2012? Catálogo de la Colección de Documentos Antiguos de la Estancia de Jesús María - Museo Jesuítico Nacional. EJMMJN-Municipalidad, Jesús María.

Foucault, M.

1985 La arqueología del saber. Siglo XXI Editores. Buenos Aires.

Gamero Rojas, M.

2011 "Repartos de tierras y plantaciones de heredades en la Carmona del Quinientos", en Carmona 7000 años de Historia Rural, dirigido por M. González Jiménez. Ayuntamiento de Carmona. Carmona.

Garaycochea, C.

2016 "La articulación económica prehispánica del Perú sur andino", en Diálogo Andino No 49:197-207.

Giorgis, M. A., A. M. Cingolani, F. Chiarini, J. Chiapella, G. Barboza, L. Ariza Espinar, R. Morero, D. E. Gurvich, P. A. Tecco, R. Subils y M. Cabido

2011 "Composición florística del Bosque Chaqueño Serrano en la provincia de Córdoba, Argentina". Kurtziana 36: 9-43.

Giovannetti, M.

2005 "La conquista del noroeste argentino y los cultivos europeos". Fronteras de la Historia 10:253-284.

Gónzalez Navarro, C.

2008 "Los pueblos de indios de la jurisdicción cordobesa a la luz de la visita de Antonio Martines Luxan de Vargas 
(1692-1693)". En Miradas sobre la historia social en la Argentina en comienzos del siglo XXI, compilado por S. Mallo y B. Moreira, pp. 185-212. CEH Carlos Segreti y CEHAC-UNLP, Córdoba.

González Navarro, C.

2010 "El servicio personal en el extremo sur de Virreinato del Perú: el caso de Córdoba a fines del siglo XVII". Revista Andina 50:199-230.

González Navarro, C. y R. Grana

2013 Conflictividad y usos sociales en la elite encomendera de Córdoba del Tucumán (Virreinato del Perú- 1573-1700). Nuevos Mundos Mundos Nuevos https://journals.openedition. org/nuevomundo/64801

Grana, R. y M.L. López

2021 "Condiciones para una práctica: El recurso cárnico en la Alimentación durante la Colonia (Siglos XVI-XVII) en el paraje Guayascate, Córdoba, Argentina". Fronteras de la Historia 26(2). En prensa.

Gruzinski, S.

2007 El pensamiento mestizo. Cultura amerindia y civilización del Renacimiento. Paidós. Barcelona.

Korstanje, A. (compil.)

2015 Crónicas materiales precolombinas: arqueología de los primeros poblados del Noroeste Argentino. Sociedad Argentina de Antropología, Buenos Aires.

Lara, M., Meneses, V. y P. de Mass 2006 "Vid, viñas y vinos en la legislación indiana: el corregimiento de Cuyo". Revista Universum 21(2):84-98.

Lema, $\mathrm{V}$.

2014 "Boceto para un esquema: domesticación y agricultura temprana en el Noroeste Argentino". Revista Española de Antropología Americana 44(2): 465-494.

Lopez, M.L.

2018 "Archaeobotany in Central Argentina. Macro and micro remains at several archaeologicalsites from early Late Holocene to early Colonial Times (3000-250 B.P.)". Vegetation History and Archaeobotany 27: 219-228.

López, M.L.

2020 "Los recursos vegetales en Guayascate. Primeros resultados del análisis arqueobotánico". Comechingonia 24(3):337-347.

Lorandi, A. M.

1988 "El servicio personal como agente de desestructuración en el Tucumán colonial”. En Revista Andina (V.6, n¹).

Marschoff, M.

2014 "La sociedad virreinal en Buenos Aires: un análisis desde la cultura material y la alimentación". Revista de Indias LXXIV (260):67-100.

Medina, M., Pastor, S. y A. Recalde.

2016 "The archaeological landscape of Late Prehispanic mixed foraging and cultivation economy (Sierras of Cordoba, Argentina)". J Anthropol Archaeol 42:88-104.

Montes, A.

2008 Indígenas y Conquistadores de Córdoba. Ediciones Isquitipe, Córdoba.

Noli, E.

1999 "La recolección en la Economía de subsistencia de las poblaciones indígenas: Una aproximación a través de fuentes coloniales (Piedemonte y Llanura Tucumano-Santiagueña, Gobernación del Tucumán)", en En los tres reinos: Prácticas de Recolección en el Cono Sur de América, editado por C.
Aschero, M. Korstanje y P. Vouto, pp. 205-215. Ediciones Magna Publicaciones. Tucumán.

Palomeque, $\mathrm{S}$.

2005 Córdoba Colonial, Economía y Sociedad. Conferencia del 6 de julio de 2005, Museo San Alberto, Córdoba.

Pastor, S. y R. Boixadós

2016 "Arqueología y etnohistoria: diálogos renovados en torno a las relaciones entre las sociedades de los llanos riojanos y de las sierras noroccidentales de córdoba (períodos prehispánico tardío y colonial temprano)", en Diálogo Andino, $\mathrm{N}^{\circ}$ 49:311-328.

Pastor, S. y M.L. López

2011 "Consideraciones sobre la agricultura prehispánica en el sector central de las Sierras de Córdoba”, en Arqueología de la agricultura: casos de estudio en la región andina argentina, editado por A. Korstanje y M. Quesada, pp. 208233. Editorial Magna, Tucumán.

Pastor, S. y M. Medina

2013 "Prácticas de resistencia, elusión y reproducción social en un contexto histórico adverso. Una mirada a los indígenas de Córdoba (Argentina) en tiempos coloniales tempranos". Memoria Americana 21(1):65-92.

Pastor S, López M.L. y D. Rivero

2012 "Access to maize (Zea mays) and its manipulation in hunter-gatherer context in central Argentina (ca. 3,0002,500 bp)". Before Farm Archaeol Anthropol Hunt Gather 2012/4:1-10.

Paz Sánchez, M.

2013 "El trigo de los pobres. La recepción del maíz en el Viejo Mundo". Revista Batey V (5):142-174.

Petrucci, N., Lema, V. Pochettino, M., Palamarczuk, V., Spano, R. y M. Tarragó

2018 "From weeds to wheat: a diachronic approach to ancient biocultural diversity in the Santa María valley, northwest Argentina". Vegetation History and Archaeobotany 27(1):229-239.

Piana, J.

1992 Los indígenas de Córdoba bajo el régimen colonial, 1570-1620. Dirección General de Publicaciones de la Universidad Nacional de Córdoba, Córdoba.

Pochettino, M.L.

2015 Botánica Económica. Las plantas interpretadas según tiempo, espacio y cultura. Sociedad Argentina de Botánica, Buenos Aires.

Rivera Medina, A.

2006 "El vino como producción, mercancía y moneda en el San Juan colonial". Revista Universum 21(2):62-82.

Saur Palmieri, V.; López, M.L.; Trillo, C.

2018 "Aproximaciones etnobotánicas de las especies y prácticas de frutos nativos comestibles de la actualidad. Aportes para la interpretación del pasado prehispánico de Cerro Colorado (Córdoba, Argentina)". Boletín de la Sociedad Argentina de Botánica 53(1): 115-133.

Tell, S.

2019 “¿Una república de “indios puros”? Comunidad, autoctonía y legitimidad. Córdoba, Gobernación del Tucumán, siglo XVIII". Memoria Americana 27(1):126-148.

Twiss, $\mathrm{K}$.

2007 The archaeology of food and identity. Center for Archaeological Investigations, South Illinois University, Carbondale. 


\section{Fuentes}

AHPC (Archivo de la Provincia de Córdoba), Escribanía 1, Año 1605, Legajo 16, Expediente 4.

AHPC, Esc.1, 1605, Leg. 16, Exp. 6.
AHPC, Esc. 1, 1605, Leg. 18, Exp. 2.

AHPC, Esc. 1, 1611, Leg. 25, Exp. 4.

AHPC, Esc. 1, 1670, Leg. 133, Exp. 7.

\section{Nota}

1 Consideramos que la ingesta se relaciona con un acto social donde se involucran diferentes actores, recursos y preparaciones en un entramado de dinámicas especialmente gestadas al interior del grupo: los sujetos dan significado a sus prácticas a la vez que se generan procesos de identificación individual y grupal de los comensales. Todo ello se engloba como Comensalismo en este manuscrito. 